\section{Critical mass in diabetes}

\section{By Lauren Martz, Staff Writer}

A University of Bremen group has found a new target-serine/ threonine kinase 4 -that can increase $\beta$ cell mass and could represent an alternative to invasive islet cell transplants in patients with diabetes. ${ }^{1}$ Next, the team will need to rule out long-term safety issues associated with blocking the kinase.

Destruction of insulin-secreting $\beta$ cells occurs in both type 1 and type 2 diabetes but through different mechanisms. In type 1 disease, an autoimmune reaction destroys the pancreatic $\beta$ cells. In type 2 , exhaustion and cellular toxicity are responsible.

Previous clinical studies have suggested that even a small amount of endogenous insulin production can maintain functional $\beta$ cells and improve patient outcomes. In patients with type 1 diabetes, intensive insulin therapy helps sustain endogenous insulin secretion and decreases the risk of chronic complications. ${ }^{2}$

Another approach in clinical development for patients with type 1 diabetes is islet cell transplants. The problems with the approach are the invasiveness of the procedure and low donor cell survival.

A team led by Kathrin Maedler and Amin Ardestani opted for a different approach-blocking the apoptosis of $\beta$ cells. The group started with a central player in multiple apoptotic processes-serine/threonine kinase 4 (STK4).

Maedler is head of the islet biology laboratory group at the University of Bremen. Ardestani is junior group leader of the islet biology laboratory group at the university. The paper also included researchers from the Lille 2 University of Health and Law, Novo Nordisk A/S, Fudan University and the University of Illinois at Chicago.

The first question was whether STK4 activation had any correlation with $\beta$ cell apoptosis.

The group found that STK4 activity was upregulated in rat $\beta$ cells and human and mouse islets under diabetogenic conditions including high glucose levels. The team also saw STK4 activation in islets from humans with type 2 diabetes and from obese, diabetic mice.

To determine the effects of STK4 activity in diabetes, the team overexpressed the kinase in human islets and rat $\beta$ cells. Adenoviral overexpression of STK4 induced $\beta$ cell apoptosis, whereas overexpression of a mutant version that had no kinase activity had no such effects.

Decreasing STK4 expression protected human islets from apoptosis and improved glucose-stimulated insulin secretion.

Notably, the improvement in insulin secretion could not be completely explained by the increase in $\beta$ cells. Overexpression of STK4 resulted in a complete loss of glucose-stimulated insulin secretion despite an incomplete loss of $\beta$ cells, suggesting another STK4dependent mechanism was at work.

STK4 overexpression also decreased levels of pancreatic and duodenal homeobox 1 (PDX1; IPF1), a transcription factor involved in $\beta$ cell development and function, compared with GFP overexpression. Decreases in PDX1 are known to alter insulin production and secretion. ${ }^{3,4}$

Thus, STK4 inhibition may help treat diabetes through two mechanisms: preventing $\beta$ cell apoptosis and restoring insulin production and secretion from the cells.

Finally, the team found that blocking Stk 4 activity helped treat mouse models of both type 1 and type 2 diabetes.

In mice with type 1 disease, $\beta$ cell-specific Stk 4 knockout improved glucose tolerance and preserved $\mathrm{Pdx} 1$ function, and it increased $\beta$ cell mass by increasing proliferation and decreasing apoptosis compared with no alteration. Systemic Stk4 knockout had similar antidiabetic effects and a similar safety profile.

In a mouse model of high-fat diet-induced type 2 diabetes, $\beta$ cellspecific knockout of $S t k 4$ also increased $\beta$ cell mass and prevented impairments in insulin secretion.

Data were published in Nature Medicine.

"The major advantage of STK4 as a drug target is that it harbors the potential to directly interfere with $\beta$ cell death and dysfunction in type 1 and type 2 diabetes. This would be a considerable advancement over currently available therapeutics that do not address the progressive loss of $\beta$ cells in type 1 and type 2 diabetes," said Cord Dohrmann, CSO of Evotec AG.

Evotec and the MedImmune LLC unit of AstraZeneca plc are developing EVT 770, a $\beta$ cell regeneration factor that is in preclinical testing to treat diabetes.

The biotech has ongoing collaborations with Harvard University and other pharmaceutical companies that are primarily targeting $\beta$ cell regenerative mechanisms.

Ole Madsen, senior principal scientist at Novo Nordisk and an honorary professor in diabetes stem cell research at the University of Copenhagen, noted that the $\beta$ cell preservation effect of inhibiting Stk4 was highly potent in mice.

Novo Nordisk markets several diabetes drugs including the longacting insulin analogs Tresiba insulin degludec and Levemir insulin detemir and the long-acting glucagon-like peptide-1 (GLP-1) agonist Victoza liraglutide.

In addition to preventing $\beta$ cell apoptosis in both type 1 and type 2 diabetes, Madsen said that another advantage of inhibiting STK4 is the potential to prevent development of the disease in patients at high risk of type 1 diabetes and in the metabolic syndrome stages of type 2 diabetes.

\section{STKler for specificity}

The challenge will be developing a kinase- and tissue-specific approach to target STK4.

Thomas Frogne, senior scientist of incretin and islet biology at Novo Nordisk and coauthor on the paper, said that there were no obvious side effects caused by global or $\beta$ cell-specific Stk 4 knockout, which is a good 


\section{ANALYSIS}

\section{"Reduced STK4 activity not only can improve cell proliferation but also has been shown to facilitate cancer development, which has to be carefully analyzed and excluded in chronic studies using animal models with global or tissue- specific knockout of STK4 before such treatment can be contemplated." \\ - George Kunos, National Institutes of Health}

indicator that inhibiting the kinase may be safe. However, he cautioned that STK4 "is widely expressed, and its global inhibition could be associated with tissue-selective side effects."

Frogne said that the company has no immediate plans to explore other STK4 inhibitors.

Dohrmann said that a challenge associated with disease-modifying strategies focused on antiapoptotic pathways is separating the positive effects from the potential safety issues associated with targeting genes involved in tumor suppression.

"In such cases, it would be of great benefit to selectively target the pancreas or ideally the $\beta$ cells," Dohrmann added.

George Kunos, a senior investigator at the NIH, wanted to see the long-term effects of STK4 inhibition. "Reduced STK4 activity not only can improve cell proliferation but also has been shown to facilitate cancer development, which has to be carefully analyzed and excluded in chronic studies using animal models with global or tissue-specific knockout of STK4 before such treatment can be contemplated," he said. The patent and licensing status of the work is undisclosed.

Martz, L. SciBX 7(13); doi:10.1038/scibx.2014.360

Published online April 3, 2014

\section{REFERENCES}

1. Ardestani, A. et al. Nat. Med.; published online March 16, 2014; doi:10.1038/nm.3482

Contact: Kathrin Maedler, University of Bremen, Bremen, Germany e-mail: kmaedler@uni-bremen.de

Contact: Amin Ardestani, same affiliation as above e-mail: ardestani.amin@gmail.com

2. The Diabetes Control and Complications Trial Research Group. Ann. Intern. Med. 128, 517-523 (1998)

3. Johnson, J.D. et al. J. Clin. Invest. 111, 1147-1160 (2003)

4. Brissova, M. et al. J. Biol. Chem. 277, 11225-11232 (2002)

\section{COMPANIES AND INSTITUTIONS MENTIONED}

AstraZeneca plc (LSE:AZN; NYSE:AZN), London, U.K.

Evotec AG (Xetra:EVT), Hamburg, Germany

Fudan University, Shanghai, China

Harvard University, Cambridge, Mass.

Lille 2 University of Health and Law, Lille, France

Medlmmune LLC, Gaithersburg, Md.

National Institutes of Health, Bethesda, Md.

Novo Nordisk A/S (CSE:NVO; NYSE:NVO), Bagsvaerd, Denmark

University of Bremen, Bremen, Germany

University of Copenhagen, Copenhagen, Denmark

University of Illinois at Chicago, Chicago, III. 